\title{
Merits of Adding Coronal-STIR Sequence in a Routine Lumbo-Sacral Spine MR Imaging Protocol to Investigate Sciatica and Sciatica-Like Symptoms
}

\author{
Medhat Refaat, Ahmed A.Torky, Mahmoud Reda
}

\author{
Department of radiology, Benha \\ faculty of medicine, Benha \\ University, Egypt \\ Correspondence to: Mahmoud \\ Reda, Department of radiology, \\ Benha faculty of medicine, \\ Benha university, Egypt \\ Email:
}

mahmoudredabenha@gmail.com

Received: 5May 2020

Accepted: 8 June 2020

\begin{abstract}
:
Background: Sciatica is defined by low back pain that radiates to the legs, with or without neurologic deficiency. sciatica or sciatica-like symptoms may result from spinal root compression in an extra-spinal location or from injury to the pelvic girdle. Aim: determine the value of coronal STIR sequence in the detection and diagnosis of extraspinal causes of sciatica by adding it to routine MRI protocol of the lumbo-sacral spine in patients referred for sciatica-like symptoms. Methods: The current study involved an observational analysis for lumbo-sacral MRI examinations done for 50 patients, 22 males and 28 females, presenting with sciatica and sciatica-like symptoms. Results and conclusion: By adding a coronal STIR sequence to the routine MRI protocol, we were able to identify extra-spinal abnormalities that were not visible on routine sequences, in 10 patients (20\%). $6(8 \%)$ patients had abnormalities which were considered to be as a definite cause of sciatica and sciatica-like symptoms. In 4 (8\%) patients, the coronal STIR also identified soft tissue abnormalities in the pelvis which were considered as equivocal causes of sciatica and sciaticalike symptoms. A higher prevalence of definite causes of extra-spinal sciatica was seen in patients between 24-40 years old. Also, prevalence of definite causes of sciatic pain was affected by history of neoplasia. Prevalence of definite causes of sciatic pain was not significantly affected by sex. However, most of the equivocal causes of sciatica were gynecological pathologies.
\end{abstract}

Keywords: coronal-STIR; umbo-sacral spine; sciatica 


\section{Introduction}

Sciatica is a common symptom, which is defined by low back pain that radiates to the legs, with or without neurologic deficiency. Similarly, in clinical practice, buttocks pain, anterior thigh pain, and claudication are often suspected to result from injury of the nerve roots in the lumbar spine (1).

Approximately half of the people eventually experience sciatica during their life period. The majority of these sciatica cases are caused by lumbar nerve root compression secondary to discogenic disease. In contrast, physicians occasionally encounter patients who present with sciatic pain, but show no radiographic evidence of lumbar nerve root compression. Such conditions have been sporadically reported in the literature as "extra-spinal sciatica" where pathologies in lumbo-sacral plexus or peripheral nerve are suggested to be involved (2).

Extra-spinal causes of sciatic pain are usually overlooked because they are rare and due to intra-spinal causes (lumbar spinal stenosis, facet joint osteoarthritis, fracture, and tumors of the spinal cord and spinal column) being the main consideration. Traumatic, infective, inflammatory, tumoral, vascular, gynecological and other causes constitute the etiology of extra-spinal sciatica. Early diagnosis of sciatica significantly improves the likelihood of relieving symptoms, as well as avoiding any additional neurologic injury and unnecessary surgery (3).

Magnetic resonance imaging (MRI), is usually the modality of choice when investigating sciatica. The recommended protocol includes sagittal views of the lumbar spine with additional axial views dedicated to lower spine disks, all of which are highly accurate in depicting disco-radicular impingement. Nevertheless, these protocols focus only on the lumbar spine and do not provide any information on the lumbo-sacral plexus or neighboring pelvic girdle, in which the extra-spinal causes of sciatica may be seen. Misdiagnosis may lead to delayed treatment and poorer outcome (4).

In this study, a coronal STIR sequence dedicated to the lumbar-pelvic-femoral area was added to routine axial and sagittal T1WI and T2WI MRI protocol of sciatica-like symptoms to investigate the bony pelvic girdle and surrounding soft tissues, including the lumbo-sacral plexus and branches. We sought to determine the value of this added sequence in evaluation of extra-spinal causes of sciatica 


\section{Patients and methods}

We performed an observational analysis for lumbo-sacral MRI examinations done for 50 patients presenting with sciatica and sciaticalike symptoms.

This cross-sectional observational study, was conducted in Benha University hospitals, between April to December 2019. Written informed consent was taken before the start of the study and was approved by Ethics Committee for Human Research of Benha faculty of medicine.

The study included 50 patients; 22 males and 28 females, with a mean age of 42.26 years (range, 24-70 years). All the patients were examined by a clinician at the outpatient clinic. The patients presenting with sciatica or sciatica-like symptoms as non-radiating low back pain, leg pain and buttock pain were included in our study. We interviewed the patients at our department to record relevant data including their age, sex, pain analysis and history of trauma, neoplasm, systemic disease or medications. We excluded patients who had undergone a lumbar spine surgery or who have a documented recent spine fracture.

All MR examinations were performed on a 1.5 Tesla MRI scanner (Aera, Siemens Medical Systems). The patient lays supine, head first. The examinations are done using a dedicated spine coil. Cushions are used for immobilization and extra-comfort for legs. The laser beam localizer is centered over the mid-abdomen (4 inches above the iliac crest).

\section{MRI protocol:}

\section{a) Routine MR Protocol:}

The protocol consisted of sagittal T1W (slice thickness, $4 \mathrm{~mm}$; FOV, $280 \mathrm{~mm}$; matrix, 307 $\times$ 384; TR/ TE, 400/8), sagittal T2W (slice thickness, $4 \mathrm{~mm}$; FOV, $280 \mathrm{~mm}$; matrix, 280 $\times$ 384; TR/TE, 3000/120) and axial T2W (slice thickness, $4 \mathrm{~mm}$; FOV, $230 \mathrm{~mm}$; matrix, $256 \times 256$; TR/TE, 3000/120). Axial images were performed at selected levels chosen from the sagittal sequences, angled through the intervertebral discs.

\section{b) Additional coronal STIR sequence:}

An additional view of the para-spinal and pelvic areas using a coronal STIR sequence dedicated to the lumbo-sacral plexus was systematically performed, ranging from the upper abdominal cavity to the lesser trochanter (slice thickness, $6 \mathrm{~mm}$; FOV, 500 mm; matrix, $269 \times 384$; TR/TE, 2500/40; TI, $150)$.

\section{Images interpretation:}

We evaluated (a) routine set of images: sagittal T1 and T2 and axial T2 images (b) additional coronal STIR images on Paxera 
Viewer. Paxera Viewer is a Cross-Platform DICOM viewer for viewing and manipulating DICOM images \& cine. The software has the ability to open and display studies obtained from different imaging modalities, apply some image processing and measurements on the image. The obtained MR sequences were evaluated by an experienced radiologist. First, the reader evaluated the routine sagittal and axial images only, and then he evaluated all sequences including the coronal STIR sequence.

\section{Statistical Analysis}

Statistical analyses were performed using the statistical package SPSS (Statistical Package for the Social Sciences). The chi-square test was applied to correlate the presence of extraspinal causes of sciatica with various data such as subgroups of age, sex, history of trauma, history of neoplasia, presence of any spinal abnormality and presence of a spinal abnormality with nerve root impingement. The exact test was used instead when the expected frequency is less than 5. P-values less than 0.05 were considered as statistically significant.

\section{Results}

Further analysis for the abnormal findings: the diagnoses by routine MRI were categorized into: normal in $9(18 \%)$ patients and spinal abnormalities in 41 (82\%). By addition of the coronal STIR sequence, the diagnosis include four categories: normal in 3 (6\%) patients, spinal abnormalities only in 37 (74\%) patients, extra-spinal abnormalities only in $6(12 \%)$ patients and both spinal and extra-spinal abnormalities in $4(8 \%)$ patients (table 1).

The spinal abnormalities were seen in 41 patients, including: disc bulge/herniation without neuro-compromise in 17 patients, disc bulge/herniation with neuro-compromise in 26 patients, facetal arthropathy in 11 patients and spondylolisthesis in 7 patients (table 2)

The coronal STIR sequence identified extraspinal abnormalities that were not visible on routine sequences in 10 patients (20\%) (table 3)

The extra-spinal abnormalities were categorized according to their relation to patients' pain into three groups; related to patients' pain, equivocal and unrelated to patients' pain; The extra-spinal abnormalities were considered to be related to sciatica in 4 patients (8\%). Identification of these abnormalities was the primary focus of the additional STIR sequence. They included: Bilateral sacroiliitis (4 patients), iliac wing metastasis (1 patient) and muscular strain (1 patient), (table 4). 
Correlation of the presence of these The prevalence of an extra-spinal cause of abnormalities with age of patients proved pain was not significantly affected by $\operatorname{sex}(\mathrm{P}=$ relevant in the age subgroup between 24-40 0.7). Conversely, it was affected by history of years old to emphasize extra-spinal cause of neoplasia $(\mathrm{P}=0.04) \quad($ table 6$)$. sciatica $(P=0.04)($ table 5$)$. Mean age was 42.26 with standard deviation 10.7 .

Table 1: Distribution of finding in routine MRI only Vs. routine \& coronal

\begin{tabular}{lll}
\hline & Routine MRI & Routine \&Coronal STIR \\
\hline Normal & 9 & 3 \\
Intra-spinal lesions & 41 & 37 \\
Extra-spinal lesions & 0 & 6 \\
Both & 0 & 4 \\
\hline
\end{tabular}

Table 2: Spinal abnormalities

\begin{tabular}{ll}
\hline Spinal abnormalities & Number of patients \\
\hline Disc lesion with neural compromise & 26 \\
Disc lesion without neural compromise & 17 \\
Spondylolisthesis & 7 \\
facet arthropathy & 11 \\
\hline
\end{tabular}

Table 3: Distribution of extra-spinal abnormalities

\begin{tabular}{lll}
\hline & Relation to pain & Number of patients \\
\hline Sacroiliitis & Related & 2 \\
Iliac lytic lesion & Related & 1 \\
Gluteal muscle strain & Related & 1 \\
Enlarged prostate & Unrelated & 2 \\
Ovarian cyst & Equivocal & 1 \\
Fibroid & Equivocal & 2 \\
Retroverted uterus & Equivocal & 1 \\
\hline
\end{tabular}


Table 4: Extra-spinal lesions related to patient's pain detected on coronal STIR

\begin{tabular}{|c|c|c|c|c|c|c|c|}
\hline patient & Sex & Age & symptom & $\begin{array}{l}\text { Associated } \\
\text { history }\end{array}$ & $\begin{array}{l}\text { Spinal abn } \\
\text { Abnormality }\end{array}$ & $\begin{array}{l}\text { mality } \\
\text { neural } \\
\text { compromise }\end{array}$ & $\begin{array}{l}\text { Extra-spinal } \\
\text { abnormality }\end{array}$ \\
\hline 1 & Male & 28 & $\begin{array}{l}\text { Low back } \\
\text { pain }\end{array}$ & None & Normal & No & Sacroiliitis \\
\hline 2 & Male & 52 & $\begin{array}{l}\text { Right } \\
\text { sciatica }\end{array}$ & $\begin{array}{l}\text { Primary } \\
\text { neoplasm }\end{array}$ & Disc protrusion & Yes & $\begin{array}{l}\text { Iliac wing } \\
\text { metastasis }\end{array}$ \\
\hline 3 & Female & 32 & $\begin{array}{l}\text { Low back } \\
\text { pain }\end{array}$ & None & Normal & No & Sacroiliitis \\
\hline 4 & Female & 45 & Left sciatica & trauma & Disc bulge & No & $\begin{array}{l}\text { Left gluteal } \\
\text { muscle strain }\end{array}$ \\
\hline
\end{tabular}

Table 5: Prevalence of extra-spinal abnormalities by age subgroups

\begin{tabular}{llll}
\hline Age subgroup & Number & with extra-spinal abnormalities & P-value \\
\hline $\mathbf{2 4 - 4 0}$ years & 29 & 5 & $0.04^{*}$ \\
$\mathbf{4 1 - 5 5}$ years & 14 & 3 & 0.1 \\
More than 56 years & 7 & 2 & 0.5 \\
\hline
\end{tabular}

Table 6: Prevalence of extra-spinal abnormalities among other features

\begin{tabular}{llll}
\hline Parameter & Number & With extra-spinal lesions & P-value \\
\hline Male & 22 & 4 & 0.7 \\
Sex Female & 28 & 6 & 0.5 \\
H/O of trauma & 3 & 1 & \\
H/O of primary neoplasm & 1 & 1 & $0.04^{*}$ \\
$\begin{array}{l}\text { Disc lesions with neural } \\
\text { compromise }\end{array}$ & 26 & 3 & 0.1 \\
\hline
\end{tabular}




\section{Case Presentation}

\section{Case 1:}

- Clinical: A male patient, 28 years old, complaining of low back pain

- Findings at routine MRI protocol: Normal (Fig. 1a).
- Findings at coronal STIR sequence: Bilateral subchondral edema in the inferior aspects of the sacroiliac joints related to bilateral sacroiliitis (Fig. $1 b$ ).

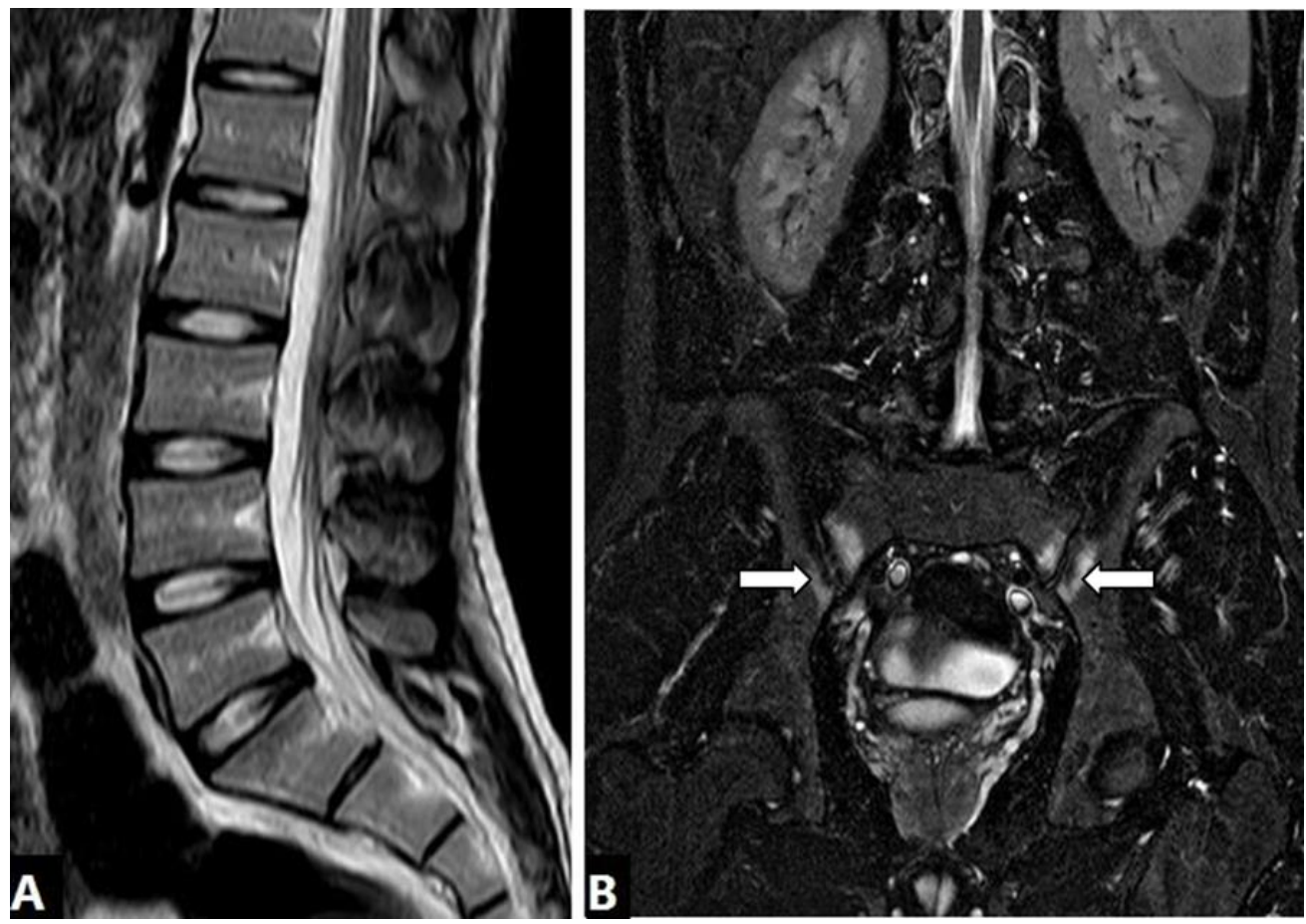

Fig.1 : (A) Sagittal T2W image showing no significant abnormality. (B) Coronal STIR image shows bilateral symmetrical edema in the subchondral and inferior aspects of the sacroiliac joints related to bilateral sacroiliitis (white arrows

\section{Case 2}

- Clinical: A male patient, 52 years old, complaining of right sciatic pain and history of pancreatic neuro-endocrine tumor.

- Findings at routine MRI protocol: Mild degenerative changes with L5/S1 tiny central disc protrusion, yet no significant neural compromise (Fig. 2a).

- Findings at coronal STIR sequence: An area of mild hyperintensity at the lower part of right iliac wing consistent with metastasis from the primary tumor (Fig. $2 b)$. 


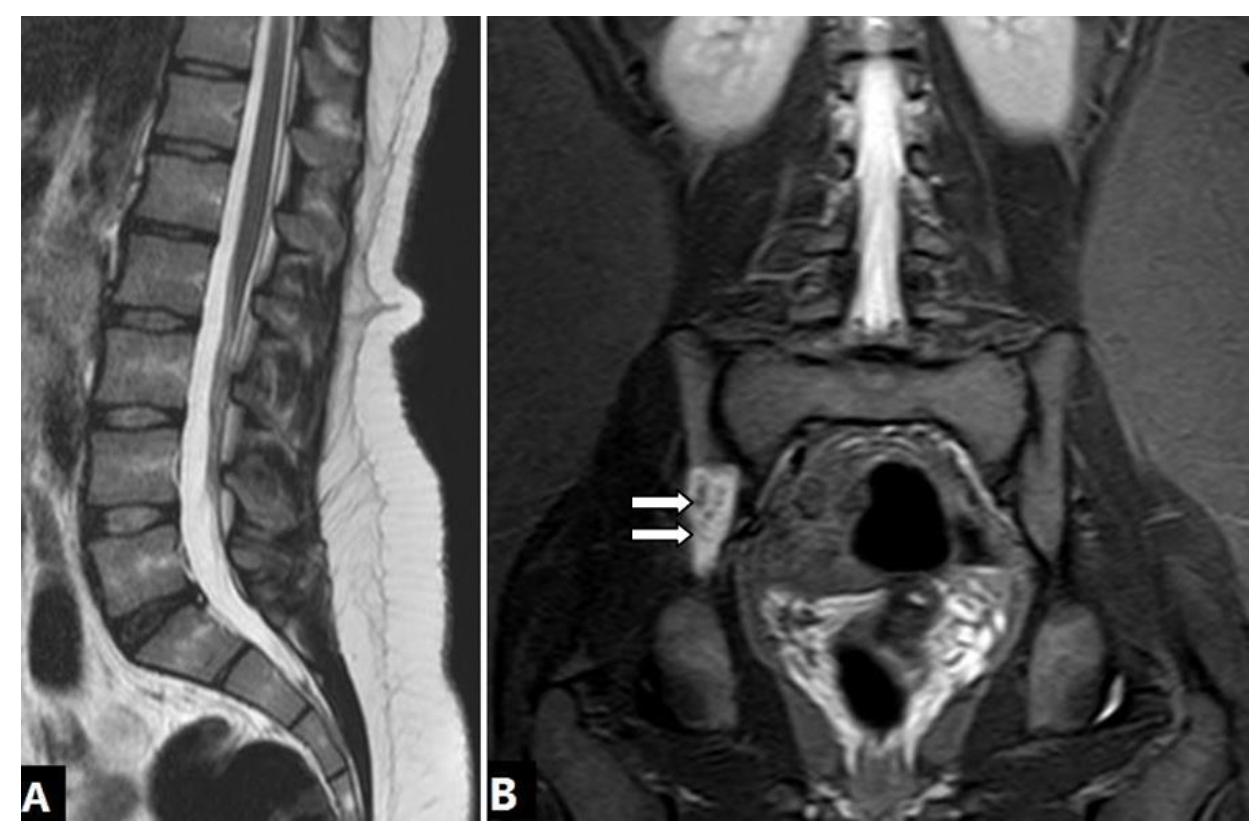

Fig. 2: (A) Sagittal T2W image showing mild degenerative changes with L5/S1 tiny central disc protrusion, yet no significant neural compromise. (B) Coronal STIR image shows area of mild hyperintensity at the lower part of right iliac wing (arrows) consistent with metastasis from the primary tumor.

\section{Discussion}

In the current study, out of the 50 patients included, 9 (18\%) patients were considered normal in the routine protocol and $3(3 \%)$ patients as normal after combining the routine protocol with the coronal-STIR sequence.

Gleeson et al., (5) showed that when sciatica was present, adding a coronal oblique STIR sequence dedicated to the sacrum and sacroiliac joint to routine MRI investigation of the lumbar spine altered the diagnosis in only $2.4 \%$ of the patients. The higher percentage in our study (8\%) compared with Gleeson et al.'s study, (5), which focused only on the sacrum and sacroiliac joints, can be explained by the use of a wider FOV in the current study. Using a larger FOV in our study including the lumbo-sacral plexus, the whole pelvis, and both hip joints, allowed the depiction of a wider spectrum of the extra-spinal abnormalities.

In correlation with previous similar studies, using the same FOV, this percentage was close to the percentage in the study of Mittal et al., 2015 study (6.8\% of 350 patients) and in the study of Laporte et al., (4) (6\% of 209 patients). 
In our study, two patients of unexpected sacroiliitis were revealed by the coronal STIR sequence. These patients belonged to the young age group and on the routine sagittal and axial images; both of them were considered to be normal without discoradicular impingement. The sciatica-like symptoms due to sacroiliitis may result from two different mechanisms: first, the sacroiliac joint may generate referred pain to the lower limbs because they share the same somatic innervation area; second, owing to the close relationship between the ventral capsule of the SI joint and the spinal nerves L5 and S1 (6). The diagnosis of sacroiliitis is frequently unreliable on clinical grounds (7). The involvement of the sacroiliac joint is considered a hallmark for the diagnosis of seronegative spondylo-arthropathy and is usually the first manifestation of this condition. Thus, the detection of sacroiliitis helps in early diagnosis and treatment (8).

Bone tumors, primary or metastatic, located either in the pelvis or at the proximal femur can cause extra-spinal sciatica. Previous history of cancer in patients with sciatica, even cancers of uncommon bone metastases, should always be a red flag; where late metastatic lesions from parotid carcinoma, cancer of uncommon bone metastases, have been reported as an extra-spinal cause of sciatica ten years after treatment of primary disease (9).
In our study, a patient with a history of primary neoplasm was diagnosed with right iliac wing bony lytic lesion, the routine MRI sequences of lumbar spine showed disc protrusion with no evidence of pelvic lesions, whereas the coronal STIR sequence revealed right iliac wing lytic lesion.

Muscle strain, especially in gluteal muscles, is an extra-spinal cause of sciatica, which can be diagnosed with coronal STIR imaging (10). In our study, the coronal STIR imaging of a patient referred with low back pain revealed gluteal muscle sprain actually responsible for the symptoms.

We found that no significant correlation was established between the presence of an extraspinal cause of sciatica and the patient's sex $(\mathrm{P}=0.7)$. These findings go in line with Laporte et al., (4).

However, our study was not in agreement with Laporte et al., study regarding the correlation of the presence of extra-spinal causes of sciatica with age and history of neoplasia. In our study, prevalence of an extra-spinal cause of pain was significantly high in patients between 24-40 years old ( $\mathrm{P}=$ 0.04) while in Laporte et al., study, a higher prevalence $(\mathrm{p}=0.029)$ was seen in patients 60 years old or older (4).

Also in our study, history of neoplasia was statistically associated with an extra-spinal 
cause of sciatica $(\mathrm{P}=0.04)$, whereas no association was established between the presence of an extra-spinal cause of sciatica and history of neoplasia in Laporte et al., 2014 study (4).

In our study, we also correlated the presence of extra-spinal causes of sciatica with history of trauma and presence of any spinal abnormality. Prevalence of an extra-spinal cause of sciatica was not significantly correlated with history of trauma $(\mathrm{P}=0.5)$.

In $6(12 \%)$ patients, the coronal STIR in our study also identified soft tissue abnormalities in the pelvis which may or may not be the cause of patient's pain. The mechanism by which these abnormalities can cause sciatica is the direct compression on the lumbo-sacral plexus and branches. Because the additional STIR sequence is limited in depicting entrapment phenomena on the lumbo-sacral plexus and branches, the relation of these abnormalities to patients' pain is still equivocal in our study.

Four of these abnormalities were due to gynecological pathologies (ovarian cysts in one patient, retroverted uterus in another and uterine fibroid in two patients). Some of these pathologies could potentially be identified on extended sagittal T2 sequences. A large ovarian cyst can potentially exert pressure effects on the sacral nerves, causing low back pain or sciatica. However, ovarian pathology typically causes pelvic pain (5). A retroverted uterus could also lie on the sacral nerves as they exit through the anterior sacral foramina, causing low back pain often more marked during uterine engorgement prior to menses. Uterine fibroid could also present with back pain (11).

Al-Khodairy et al. (12) reported that the search for a gynecological etiology for pain should be considered even though imaging techniques reveal either a herniated disc or other pathologic conditions of the spine which correlate with the symptoms .

The main limitation of the study is that the additional STIR sequence proposed in this study may lack sensitivity in depicting small neuromas or entrapment phenomena on the lumbo-sacral plexus and branches because of its sequential pattern and high section thickness. Such visualization of nerve injury may be improved by using neurography techniques such as T2-weighted or STIR sequences with maximum- intensityprojection reconstruction or the more recently developed 3D nerve selective techniques based on diffusion-weighted sequences with directional encoding.

\section{Conclusion}

Adding a coronal STIR sequence to the routine MRI protocol is valuable to identify extra-spinal causes of sciatica and sciatica- 
like symptoms. This sequence is significantly valuable when sciatic symptoms appear in patients with any of the following features: young patients between 24-40 years old, patients with history of neoplasia. The sequence may also be valuable in female patients where some gynecological pathologies may account for sciatica-like symptoms. So, we recommend adding a coronal STIR sequence to the routine lumbo-sacral MRI when investigating sciatica and sciatica-like symptoms in patients with any of the above mentioned features because it may provide diagnostic information and patient care could be improved as a result with minimal additional cost of a slight increase in the acquisition time.

\section{Sources of funding}

This research did not receive any specific grant from funding agencies in the public, commercial, or not-for-profit sectors.

\section{References}

1. Valat J-P, Genevay S, Marty M, Rozenberg S, Koes B. Sciatica. Best Pract Res Clin Rheumatol. 2010;24(2):241-52.

2. Yoshimoto M, Kawaguchi S, Takebayashi T, Isogai $\mathrm{S}$, Kurata $\mathrm{Y}$, Nonaka $\mathrm{S}$, et al. Diagnostic features of sciatica without lumbar nerve root compression. Clin Spine Surg. 2009;22(5):328-33.
3. Ergun T, Lakadamyali H, Derincek A, Tarhan NC, Ozturk A. Magnetic resonance imaging in the visualization of benign tumors and tumor-like lesions of hand and wrist. Curr Probl Diagn Radiol. 2010;39(1):1-16.

4. Laporte C, Albert J-D, Duvauferrier R, Bertaud V, Gouillou M, Guillin R. MRI investigation of radiating pain in the lower limbs: value of an additional sequence dedicated to the lumbosacral plexus and pelvic girdle. Am J Roentgenol. 2014;203(6):1280-5.

5. Gleeson TG, O'connell MJ, Duke D, Ryan M, Ennis R, Eustace SJ. Coronal oblique turbo STIR imaging of the sacrum and sacroiliac joints at routine MR imaging of the lumbar spine. Emerg Radiol. 2005;12(1-2):38-43.

6. Buijs E, Visser L, Groen G. Sciatica and the sacroiliac joint: a forgotten concept. $\mathrm{Br} \mathrm{J}$ Anaesth 2007;99(5):713-6.

7. Jans L, Van Praet L, Elewaut D, Van den Bosch F, Carron P, Jaremko JL, et al. MRI of the SI joints commonly shows non-inflammatory disease in patients clinically suspected of sacroiliitis. Eur J Radiol. 2014;83(1):179-84.

8. Prakash D, Prabhu SM, Irodi A Seronegative spondyloarthropathy-related sacroiliitis: CT, MRI features and differentials. Indian J Radiol Imaging. 2014;24(3):271.

9. Panagiotopoulos EC. Our Experience in Extra-Pelvic Causes of Sciatica and Review of Literature. 2017;

10. Gupta R, Mittal P, Mittal A, Mittal K, Gupta S, Kaur R. Additional merit of coronal STIR imaging for MR imaging of lumbar spine. $\mathrm{J}$ craniovertebral junction spine. 2015;6(1):12.

11. Murphy DR, Bender MI, Green G. Uterine fibroid mimicking lumbar radiculopathy: a case report. Spine (Phila Pa 1976). 2010;35(24):E1435-7.

12. Al-Khodairy A-WT, Bovay P, Gobelet C. Sciatica in the female patient: anatomical considerations, aetiology and review of the literature. Eur Spine J. 2007;16(6):721-31.

To cite this article: Medhat Refaat, Ahmed A.Torky, Mahmoud Reda. Merits of Adding Coronal-STIR Sequence in a Routine Lumbo-Sacral Spine MR Imaging Protocol to Investigate Sciatica and Sciatica-Like Symptoms, BMFJ 2020; 37 (Radiology): 21-31. DOI: $10.21608 / \mathrm{bmfi} .2020 .29465 .1257$ 